\title{
Synthesis of 4-Benzylidene-2,5-dimethyl-3,4-dihydro-2H-pyrrole Derivatives from Baylis-Hillman Adducts and Their Chemical Transformations
}

\author{
Mi Jung Lee, Ka Young Lee, Da Yeon Park, and Jae Nyoung Kim* \\ Department of Chemistry and Institute of Basic Science, Chonnam National University, Gwangju 500-757, Korea \\ *E-mail:kimjn@chonnam.ac.kr \\ Received June 3, 2005
}

Key Words : Baylis-Hillman adducts, Nitroalkanes, Reductive cyclization, Pyrrolines, Michael reaction

Recently, Basavaiah and Rao reported the synthesis of substituted $\gamma$-lactams by the reductive cyclization of $\gamma$ nitroesters, which were prepared from the reaction of the acetates of Baylis-Hillman adducts and nitro compounds. ${ }^{1} \gamma$ Nitrocarbonyl compounds could be transformed into cyclic nitrones or pyrroline derivatives depending upon the reduction conditions and the nature of the carbonyl groups. ${ }^{1,2}$ Various reduction conditions have been used for the reductive cyclization of $\gamma$-nitrocarbonyl compounds including $\mathrm{Fe} /$ $\mathrm{AcOH},{ }^{1} \mathrm{Zn} / \mathrm{NH}_{4} \mathrm{Cl},{ }^{2 \mathrm{a}-\mathrm{d}}$ and catalytic hydrogenation. ${ }^{2 \mathrm{e}, 2 \mathrm{f}}$

Suitably substituted pyrrolines ${ }^{3}$ or cyclic nitrone derivatives ${ }^{4}$ have been prepared and used as important synthetic intermediates. ${ }^{1-4}$ During the investigation on the chemical transformations of Baylis-Hillman adducts, ${ }^{5}$ we intended to examine the reductive cyclization of $\gamma$-nitroketone derivatives 2 derived from the acetates of Baylis-Hillman adducts as shown in Scheme 1. The starting materials 2a-e were easily prepared by the $\mathrm{S}_{N} 2$ ' reaction of primary nitroalkanes and the acetates of the Baylis-Hillman adducts according to the previous method. ${ }^{1,5}$ We tried the reductive cyclization of $\mathbf{2 a}$ under various conditions and the results are summarized in Table 1 (entries 1-3). As shown in Table 1, we obtained mixtures of 4-benzylidene-2,5-dimethyl-3,4-dihydro- $2 H$ pyrrole (5a) and cyclic nitrone derivative $\mathbf{6 a}$ in variable yields. The use of $\mathrm{Fe} / \mathrm{AcOH}$ gave the pyrroline derivative $\mathbf{5 a}$ as the major product under refluxing conditions (entry 1). Whereas, we obtained the cyclic nitrone derivative $6 \mathbf{a}$ as the major product when we use $\mathrm{Zn} / \mathrm{NH}_{4} \mathrm{Cl}$ at low temperature (entry 3). The use of $\mathrm{Fe} / \mathrm{AcOH}$ (entry 2) at lower temperature and $\mathrm{Zn} / \mathrm{NH}_{4} \mathrm{Cl}$ at room temperature (not shown) showed diminished selectivity. In spite of our extensive efforts we failed to obtain higher selectivity. Similarly, we synthesized $\mathbf{5 b} \mathbf{b}-\mathbf{e}$ and $\mathbf{6 b}-\mathbf{e}$ from the reaction of $\mathbf{2} \mathbf{b}-\mathbf{e}$ under
$\mathrm{Fe} / \mathrm{AcOH} / \mathrm{reflux}$ conditions and the results are summarized in Table 1.

For the substrates $\mathbf{2 b - d , ~ p y r r o l i n e ~ d e r i v a t i v e s ~} \mathbf{5 b - d}$ were isolated as the major products. However, nitrone $\mathbf{6 e}$ was obtained as the major product in the case of dimethylsubstituted starting material 2e (entry 7). Structure identification of the synthesized products was carried out by their ${ }^{1} \mathrm{H}$ and ${ }^{13} \mathrm{C}$ NMR, IR, mass, and chemical transformations (vide infra). The stereochemistry of the double bond of 5a and 6a was confirmed as $E$ based on NOE experiments (shown in Table 1). ${ }^{1,5}$ The mechanism for the formation of 5 and $\mathbf{6}$ was proposed as in Scheme 2 with $\mathbf{5 a}$ and $\mathbf{6 a}$ as the representative examples. Reduction of the nitro group into amino group to form 3a and the following condensation gave 5a. Partial reduction to hydroxylamine derivative $4 \mathbf{a}$ and the following cyclization and dehydration afforded $\mathbf{6 a}$.

In order to verify the usefulness of the prepared pyrroline compounds $\mathbf{5}$, we examined the Michael addition reaction of the acidic methyl group at the 5-position of $\mathbf{5 a}$ toward acrylonitrile or methyl acrylate (Scheme 3). ${ }^{6}$ The reaction of pyrroline 5a and acrylonitrile in $\mathrm{THF}$ in the presence of catalytic amounts of base (DBU or $\mathrm{NaOMe}$ ) produced intractable mixtures of products. Fortunately, we could obtain 8a in moderate yield $(59 \%)$ by refluxing 5a in acrylonitrile without any base and solvent for long time (60 h). First introduction of acrylonitrile to the methyl group of 5a produced the corresponding mono adduct (I), which reacted once more with acrylonitrile to produce $\mathbf{8 a}$. But, the third introduction of acrylonitrile to $\mathbf{8 a}$ did not occur presumably due to the steric hindrance. The Michael addition reaction was thought to occur via the imine-enamine tautomerization as shown. ${ }^{7}$ Similarly, we obtained 7a from the reaction of 5a and methyl acrylate in 58\% yield. The<smiles>C=C(C(C)=O)C(OC(C)=O)c1ccccc1</smiles>

1a

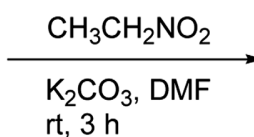

$\mathrm{rt}, 3 \mathrm{~h}$<smiles>CC(=O)/C(=C/c1ccccc1)CC(C)[N+](=O)[O-]</smiles>

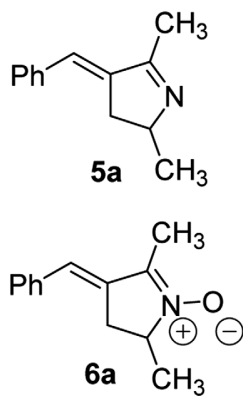


Table 1. Reductive cyclization of 2

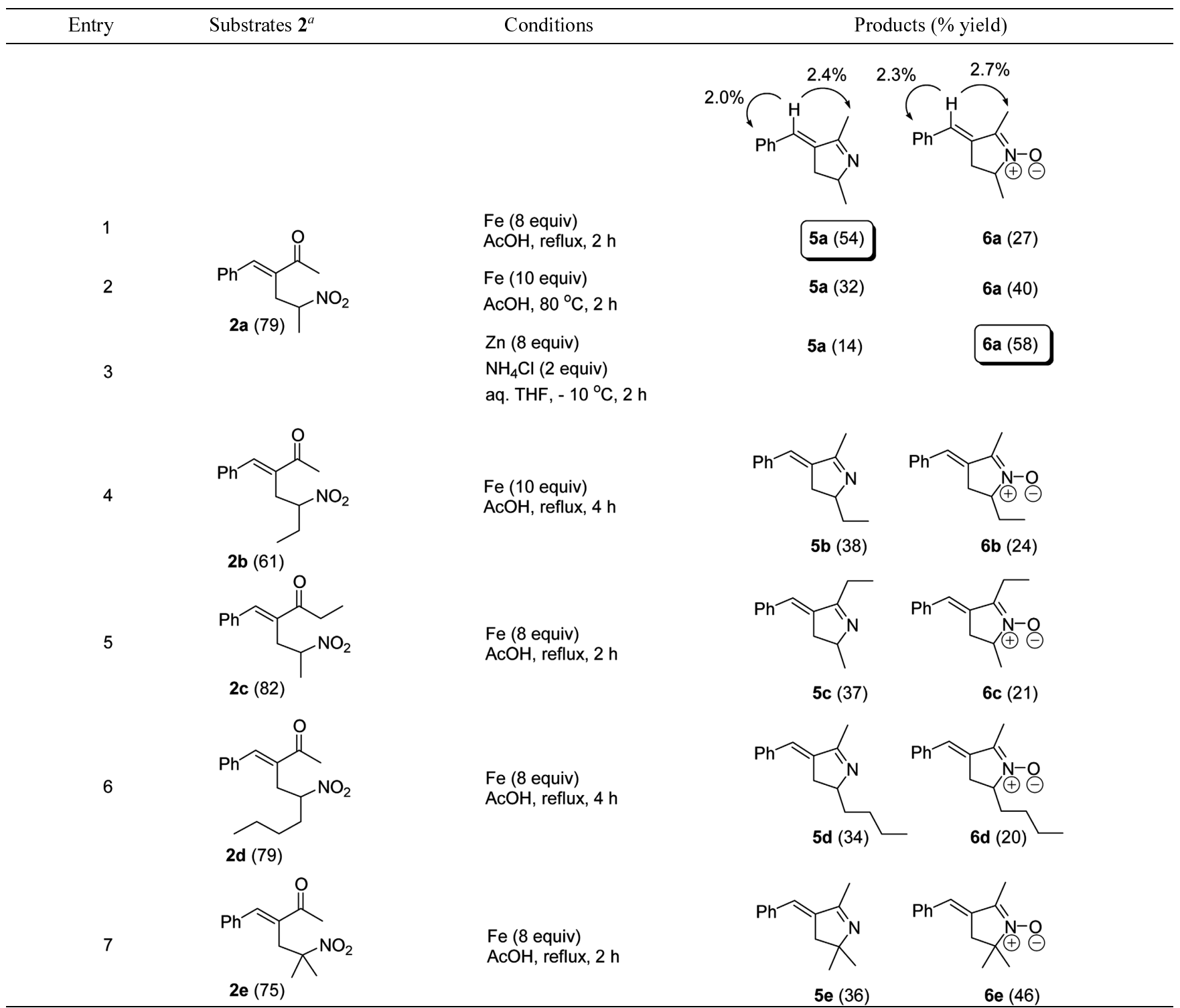

${ }^{a}$ Yields in parenthesis. Conditions: Baylis-Hillman acetate 1, nitroalkane (2 equiv), $\mathrm{K}_{2} \mathrm{CO}_{3}$ (3 equiv), DMF, $\mathrm{rt}, 3 \mathrm{~h}$.<smiles>CC(=O)/C(=C\c1ccccc1)CC(C)N(C)/C=C(\CC(C)N)C(C)=O</smiles>

Scheme 2

compound 7a could be transformed to bicyclic lactam derivative $9 \mathbf{a}$ in refluxing toluene in the presence of acetic acid in $48 \%$ yield. ${ }^{8,9}$ We also tried the reactions of $\mathbf{5 e}$ and $\mathbf{5 c}$ and obtained the corresponding tetrahydroindolizinone derivatives $\mathbf{9 b}$ and $9 \mathbf{c}$ via the corresponding intermediates 7b and 7c although the yields were relatively low (Schemes 4 and 5).

In summary, we disclosed the reductive cyclization of $\gamma$-nitrocarbonyl compounds derived from Baylis-Hillman adducts into cyclic nitrone and pyrroline derivatives. 
<smiles>CC1CC(=Cc2ccccc2)C(C(CCC#N)CCC#N)=N1</smiles><smiles>CC1CC(=Cc2ccccc2)C(CCCC#N)=N1</smiles>

(I)
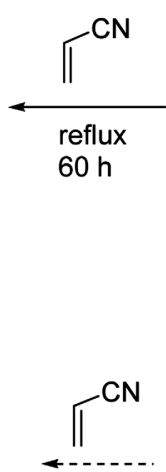<smiles>CC1=NC(C)C/C1=C\c1ccccc1</smiles>

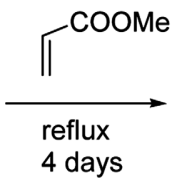<smiles>C=CC=C</smiles><smiles>C=C1NC(C)C/C1=C\c1ccccc1</smiles>

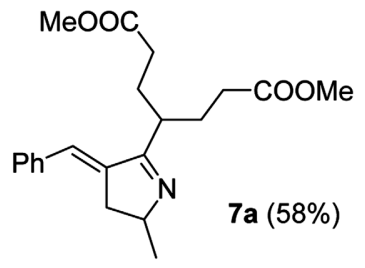

$\mathrm{AcOH}$ (6 equiv) toluene, reflux 3 days

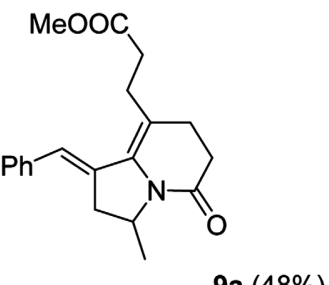

Scheme 3
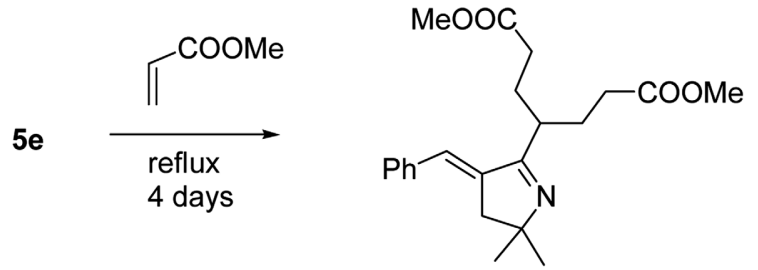

$7 b(28 \%)$

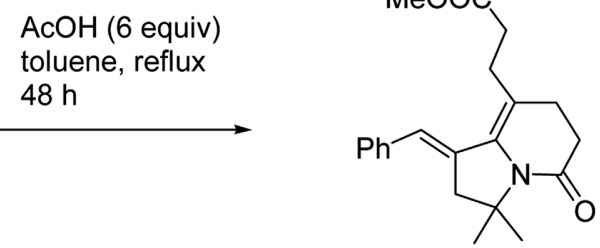

9b $(40 \%)$

Scheme 4

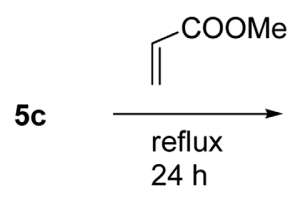<smiles>CC(=O)CCC(C)C1=NC(C)CC1=Cc1ccccc1</smiles>

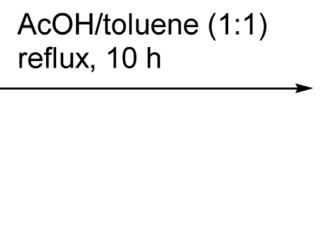

Scheme 5

Selective double Michael addition reaction of the pyrroline compounds was observed for the first time. Further studies on the synthesis of bicyclic lactam derivatives and transformation into natural alkaloid derivatives are underway. ${ }^{9}$

\section{Experimental Section}

Typical procedure for the synthesis of starting material 2a: A solution of Baylis-Hillman acetate 1a $(436 \mathrm{mg}, 2$ $\mathrm{mmol}$ ), nitroethane $(300 \mathrm{mg}, 4 \mathrm{mmol})$, and $\mathrm{K}_{2} \mathrm{CO}_{3}(830 \mathrm{mg}$, $6 \mathrm{mmol})$ in DMF ( $5 \mathrm{~mL}$ ) was stirred at room temperature for $3 \mathrm{~h}$. After the normal aqueous workup and column chromatographic purification process (hexanes/ether, $10: 1$ ) we obtained 2a, $370 \mathrm{mg}$ (79\%). Other starting materials $\mathbf{2 b - e}$ were synthesized similarly and the spectroscopic data are as follows.

Compound 2a: 79\%; oil; IR (neat) 1666, $1550 \mathrm{~cm}^{-1}$; ${ }^{1} \mathrm{H}$
$\mathrm{NMR}\left(\mathrm{CDCl}_{3}, 300 \mathrm{MHz}\right) \delta 1.44(\mathrm{~d}, J=6.6 \mathrm{~Hz}, 3 \mathrm{H}), 2.48(\mathrm{~s}$, $3 \mathrm{H}), 2.93(\mathrm{ddd}, J=14.1,5.7$, and $0.9 \mathrm{~Hz}, 1 \mathrm{H}), 3.15(\mathrm{dd}, J=$ 14.1 and $9.0 \mathrm{~Hz}, 1 \mathrm{H}), 4.77-4.89(\mathrm{~m}, 1 \mathrm{H}), 7.27-7.46(\mathrm{~m}, 5 \mathrm{H})$, $7.70(\mathrm{~s}, 1 \mathrm{H}) ;{ }^{13} \mathrm{C} \mathrm{NMR}\left(\mathrm{CDCl}_{3}, 75 \mathrm{MHz}\right) \delta 18.80,25.84$, $31.92,81.45,128.65,128.73,129.01,134.48,137.00$, $143.85,199.64$

Compound 2b: 61\%; oil; IR (neat) 1666, $1547 \mathrm{~cm}^{-1} ;{ }^{1} \mathrm{H}$ $\operatorname{NMR}\left(\mathrm{CDCl}_{3}, 300 \mathrm{MHz}\right) \delta 0.88(\mathrm{t}, J=7.2 \mathrm{~Hz}, 3 \mathrm{H}), 1.67-$ $1.76(\mathrm{~m}, 1 \mathrm{H}), 1.86-1.97(\mathrm{~m}, 1 \mathrm{H}), 2.47(\mathrm{~s}, 3 \mathrm{H}), 2.93(\mathrm{dd}, J=$ 14.1 and $4.5 \mathrm{~Hz}, 1 \mathrm{H}), 3.13(\mathrm{dd}, J=14.1$ and $9.6 \mathrm{~Hz}, 1 \mathrm{H})$, 4.60-4.71 (m, 1H), 7.28-7.44 (m, 5H), $7.69(\mathrm{~s}, 1 \mathrm{H}) ;{ }^{13} \mathrm{C}$ $\mathrm{NMR}\left(\mathrm{CDCl}_{3}, 75 \mathrm{MHz}\right) \delta 10.12,25.84,26.77,30.52,88.27$, 128.63, 128.66, 128.94, 134.47, 136.98, 143.80, 199.63.

Compound 2c: $82 \%$; oil; IR (neat) $1670,1547 \mathrm{~cm}^{-1} ;{ }^{1} \mathrm{H}$ $\mathrm{NMR}\left(\mathrm{CDCl}_{3}, 300 \mathrm{MHz}\right) \delta 1.18(\mathrm{t}, J=7.2 \mathrm{~Hz}, 3 \mathrm{H}), 1.43(\mathrm{~d}$, $J=6.7 \mathrm{~Hz}, 3 \mathrm{H}), 2.77-2.96(\mathrm{~m}, 3 \mathrm{H}), 3.11-3.19(\mathrm{~m}, 1 \mathrm{H}), 4.78-$ $4.88(\mathrm{~m}, 1 \mathrm{H}), 7.26-7.45(\mathrm{~m}, 5 \mathrm{H}), 7.71(\mathrm{~s}, 1 \mathrm{H}) ;{ }^{13} \mathrm{C} \mathrm{NMR}$ $\left(\mathrm{CDCl}_{3}, 75 \mathrm{MHz}\right) \delta 8.65,18.87,30.74,32.23,81.57,128.65$, 
128.74, 128.91, 134.64, 136.63, 142.45, 202.43.

Compound 2d: 79\%; IR (neat) $1670,1550 \mathrm{~cm}^{-1}$; ${ }^{1} \mathrm{H}$ NMR $\left(\mathrm{CDCl}_{3}, 300 \mathrm{MHz}\right) \delta 0.85(\mathrm{t}, J=7.2 \mathrm{~Hz}, 3 \mathrm{H}), 1.14-1.35(\mathrm{~m}$, $4 \mathrm{H}), 1.57-1.70(\mathrm{~m}, 1 \mathrm{H}), 1.81-1.95(\mathrm{~m}, 1 \mathrm{H}), 2.47(\mathrm{~s}, 3 \mathrm{H})$, $2.91(\mathrm{dd}, J=14.1$ and $4.8 \mathrm{~Hz}, 1 \mathrm{H}), 3.13(\mathrm{dd}, J=14.1$ and 9.6 $\mathrm{Hz}, 1 \mathrm{H}), 4.66-4.76$ (m, 1H), 7.25-7.45 (m, 5H), 7.68 (s, 1H); ${ }^{13} \mathrm{C} \mathrm{NMR}\left(\mathrm{CDCl}_{3}, 75 \mathrm{MHz}\right) \delta 13.65,21.98,25.88,27.72$, 30.82 , 33.19, 86.88, 128.65, 128.69, 128.96, 134.53, 137.08, 143.81, 199.68 .

Compound 2e: 75\%; oil; IR (neat) $1674,1539 \mathrm{~cm}^{-1} ;{ }^{1} \mathrm{H}$ $\mathrm{NMR}\left(\mathrm{CDCl}_{3}, 300 \mathrm{MHz}\right) \delta 1.37(\mathrm{~s}, 6 \mathrm{H}), 2.48(\mathrm{~s}, 3 \mathrm{H}), 3.30$ (s, 2H), 7.27-7.44 (m, 5H), $7.71(\mathrm{~s}, 1 \mathrm{H}) ;{ }^{13} \mathrm{C} \mathrm{NMR}\left(\mathrm{CDCl}_{3}\right.$, $75 \mathrm{MHz}) \delta$ 25.81, 26.00, 34.55, 87.88, 128.50, 128.75, $128.78,135.04,137.79,143.24,199.62$.

Typical procedure for the synthesis of pyrroline $5 \mathrm{a}$ and cyclic nitrone 6a: To a stirred mixture of 2a (233 mg, 1 $\mathrm{mmol})$ in $\mathrm{AcOH}(3 \mathrm{~mL})$ was added Fe powder $(447 \mathrm{mg}, 8$ mmol) and heated to reflux for $2 \mathrm{~h}$. After cooling the reaction mixtures to room temperature, dilution with ether, filtration over Celite pad, normal aqueous workup with ether, and column chromatographic purification process (hexanes $/ \mathrm{CH}_{2} \mathrm{Cl}_{2} /$ EtOAc, $2: 1: 1$ ) we obtained pyrroline 5a and nitrone 6a, $100 \mathrm{mg}(54 \%), 55 \mathrm{mg}$ (27\%), respectively. Other experiments for the synthesis of $\mathbf{5 b}$-e and $\mathbf{6 b}$-e were carried out similarly and the spectroscopic data of prepared compounds are as follows.

Compound 5a: 54\%; oil; IR (neat) 2962, $1601 \mathrm{~cm}^{-1}$; ${ }^{1} \mathrm{H}$ $\mathrm{NMR}\left(\mathrm{CDCl}_{3}, 300 \mathrm{MHz}\right) \delta 1.32(\mathrm{~d}, J=6.9 \mathrm{~Hz}, 3 \mathrm{H}), 2.22(\mathrm{~d}$, $J=1.8 \mathrm{~Hz}, 3 \mathrm{H}), 2.43(\mathrm{dt}, J=17.7$ and $3.0 \mathrm{~Hz}, 1 \mathrm{H}), 3.08$ (ddd, $J=17.7,7.2$, and $3.0 \mathrm{~Hz}, 1 \mathrm{H}), 4.18-4.28(\mathrm{~m}, 1 \mathrm{H}), 6.70$ $(\mathrm{t}, J=3.0 \mathrm{~Hz}, 1 \mathrm{H}), 7.25-7.48(\mathrm{~m}, 5 \mathrm{H}) ;{ }^{13} \mathrm{C} \mathrm{NMR}\left(\mathrm{CDCl}_{3}, 75\right.$ MHz) $\delta 16.05,22.50,37.65,65.36,124.98,127.75,128.56$, 128.71, 136.90, 143.08, 171.17; ESIMS $m / z$ 186.40 $\left(\mathrm{M}^{+}+\mathrm{H}\right)$.

Compound 6a: 27\%; white solid, mp 64-65 ${ }^{\circ} \mathrm{C}$; IR (neat) $1550,1269 \mathrm{~cm}^{-1} ;{ }^{1} \mathrm{H} \mathrm{NMR}\left(\mathrm{CDCl}_{3}, 300 \mathrm{MHz}\right) \delta 1.53(\mathrm{~d}, J=$ $6.9 \mathrm{~Hz}, 3 \mathrm{H}), 2.17$ (d, $J=1.5 \mathrm{~Hz}, 3 \mathrm{H}), 2.76$ (ddd, $J=16.5$, 3.9, and $2.4 \mathrm{~Hz}, 1 \mathrm{H}), 3.35$ (ddd, $J=16.5,8.4$, and $2.4 \mathrm{~Hz}$, $1 \mathrm{H}), 4.17-4.27(\mathrm{~m}, 1 \mathrm{H}), 6.50(\mathrm{t}, J=2.4 \mathrm{~Hz}, 1 \mathrm{H}), 7.22-7.42$ $(\mathrm{m}, 5 \mathrm{H}) ;{ }^{13} \mathrm{C} \mathrm{NMR}\left(\mathrm{CDCl}_{3}, 75 \mathrm{MHz}\right) \delta 9.22,19.54,33.39$, $66.62,122.17,127.25,128.49,128.65,135.03,136.50$, 145.27; ESIMS $m / z$ 202.06 $\left(\mathrm{M}^{+}+\mathrm{H}\right)$.

Compound 5b: 38\%; oil; IR (neat) 2958, 2924, $1601 \mathrm{~cm}^{-1}$; ${ }^{1} \mathrm{H}$ NMR $\left(\mathrm{CDCl}_{3}, 300 \mathrm{MHz}\right) \delta 1.02(\mathrm{t}, J=7.2 \mathrm{~Hz}, 3 \mathrm{H}), 1.42-$ $1.57(\mathrm{~m}, 1 \mathrm{H}), 1.72-1.87(\mathrm{~m}, 1 \mathrm{H}), 2.21$ (d, $J=1.8 \mathrm{~Hz}, 3 \mathrm{H})$, $2.47(\mathrm{dt}, J=17.7$ and $2.7 \mathrm{~Hz}, 1 \mathrm{H}), 3.00(\mathrm{ddd}, J=17.7,6.9$, and $2.7 \mathrm{~Hz}, 1 \mathrm{H}), 4.01-4.12(\mathrm{~m}, 1 \mathrm{H}), 6.68(\mathrm{t}, J=2.7 \mathrm{~Hz}, 1 \mathrm{H})$, 7.25-7.48 (m, 5H); ${ }^{13} \mathrm{C} \mathrm{NMR}\left(\mathrm{CDCl}_{3}, 75 \mathrm{MHz}\right) \delta 10.64$, $16.05,29.53,35.30,71.57,124.58,127.65,128.51,128.66$, 136.93, 142.94, 171.24 .

Compound 6b: 24\%; oil; IR (neat) 2966, 2931, 1547, 1273 $\mathrm{cm}^{-1}{ }^{1} \mathrm{H}$ NMR $\left(\mathrm{CDCl}_{3}, 300 \mathrm{MHz}\right) \delta 0.92(\mathrm{t}, J=7.2 \mathrm{~Hz}, 3 \mathrm{H})$, $1.72-1.83(\mathrm{~m}, 1 \mathrm{H}), 2.09-2.22(\mathrm{~m}, 1 \mathrm{H}), 2.18(\mathrm{~d}, J=1.2 \mathrm{~Hz}$, $3 \mathrm{H}), 2.82(\mathrm{dt}, J=16.8$ and $2.7 \mathrm{~Hz}, 1 \mathrm{H}), 3.25(\mathrm{ddd}, J=16.8$, 8.4, and $2.7 \mathrm{~Hz}, 1 \mathrm{H}), 4.09-4.18(\mathrm{~m}, 1 \mathrm{H}), 6.50(\mathrm{t}, J=2.4 \mathrm{~Hz}$, $1 \mathrm{H}), 7.23-7.40(\mathrm{~m}, 5 \mathrm{H}) ;{ }^{13} \mathrm{C} \mathrm{NMR}\left(\mathrm{CDCl}_{3}, 75 \mathrm{MHz}\right) \delta 8.36$, 9.10, 25.55, 30.49, 71.60, 122.17, 127.29, 128.54, 128.69, $135.32,136.54,146.09$.
Compound 5c: 37\%; oil; IR (neat) 2970, 1601, $1450 \mathrm{~cm}^{-1}$; ${ }^{1} \mathrm{H}$ NMR $\left(\mathrm{CDCl}_{3}, 300 \mathrm{MHz}\right) \delta 1.28(\mathrm{t}, J=7.5 \mathrm{~Hz}, 3 \mathrm{H}), 1.31$ $(\mathrm{d}, J=6.9 \mathrm{~Hz}, 3 \mathrm{H}), 2.43(\mathrm{dt}, J=17.4$ and $2.7 \mathrm{~Hz}, 1 \mathrm{H}), 2.57$ (qd, $J=7.5$ and $1.5 \mathrm{~Hz}, 2 \mathrm{H}), 3.07$ (ddd, $J=17.4,6.9$, and 2.7 $\mathrm{Hz}, 1 \mathrm{H}), 4.19-4.28(\mathrm{~m}, 1 \mathrm{H}), 6.71(\mathrm{t}, J=2.7 \mathrm{~Hz}, 1 \mathrm{H}), 7.24-$ $7.47(\mathrm{~m}, 5 \mathrm{H}) ;{ }^{13} \mathrm{C} \mathrm{NMR}\left(\mathrm{CDCl}_{3}, 75 \mathrm{MHz}\right) \delta 11.26,22.59$, 22.69, 38.01, 65.31, 124.21, 127.60, 128.50, 128.69, 136.99, $142.34,174.99$

Compound 6c: 21\%; oil; IR (neat) $1539,1273 \mathrm{~cm}^{-1} ;{ }^{1} \mathrm{H}$ $\mathrm{NMR}\left(\mathrm{CDCl}_{3}, 300 \mathrm{MHz}\right) \delta 1.20(\mathrm{t}, J=7.5 \mathrm{~Hz}, 3 \mathrm{H}), 1.52(\mathrm{~d}$, $J=6.6 \mathrm{~Hz}, 3 \mathrm{H}), 2.66(\mathrm{q}, J=7.5 \mathrm{~Hz}, 2 \mathrm{H}), 2.71-2.79(\mathrm{~m}, 1 \mathrm{H})$, 3.35 (ddd, $J=16.5,8.4$, and $2.7 \mathrm{~Hz}, 1 \mathrm{H}), 4.17-4.25(\mathrm{~m}, 1 \mathrm{H})$, $6.53(\mathrm{t}, J=2.7 \mathrm{~Hz}, 1 \mathrm{H}), 7.22-7.45(\mathrm{~m}, 5 \mathrm{H}) ;{ }^{13} \mathrm{C} \mathrm{NMR}$ $\left(\mathrm{CDCl}_{3}, 75 \mathrm{MHz}\right) \delta 9.66,16.67,19.59,33.40,66.56,121.91$, 127.24, 128.52, 128.66, 134.06, 136.59, 149.70 .

Compound 5d: 34\%; IR (neat) 2954, 2927, $1601 \mathrm{~cm}^{-1} ;{ }^{1} \mathrm{H}$ $\operatorname{NMR}\left(\mathrm{CDCl}_{3}, 300 \mathrm{MHz}\right) \delta 0.92(\mathrm{t}, J=6.9 \mathrm{~Hz}, 3 \mathrm{H}), 1.33-$ $1.47(\mathrm{~m}, 5 \mathrm{H}), 1.72-1.83(\mathrm{~m}, 1 \mathrm{H}), 2.24(\mathrm{~d}, J=1.8 \mathrm{~Hz}, 3 \mathrm{H})$, $2.49(\mathrm{dt}, J=17.4$ and $2.7 \mathrm{~Hz}, 1 \mathrm{H}), 3.01$ (ddd, $J=17.4,7.2$, and $2.7 \mathrm{~Hz}, 1 \mathrm{H}), 4.10-4.19(\mathrm{~m}, 1 \mathrm{H}), 6.72(\mathrm{t}, J=2.7 \mathrm{~Hz}, 1 \mathrm{H})$, 7.20-7.48 (m, 5H); ${ }^{13} \mathrm{C} \mathrm{NMR}\left(\mathrm{CDCl}_{3}, 75 \mathrm{MHz}\right) \delta 13.98$, $15.70,22.72,28.49,35.56,36.37,69.71,125.48,127.84$, $128.55,128.76,136.71,142.56,171.69$.

Compound 6d: 20\%; oil; IR (neat) 2954, 1550, $1269 \mathrm{~cm}^{-1}$; ${ }^{1} \mathrm{H} \mathrm{NMR}\left(\mathrm{CDCl}_{3}, 300 \mathrm{MHz}\right) \delta 0.92(\mathrm{t}, J=6.9 \mathrm{~Hz}, 3 \mathrm{H}), 1.23-$ $1.43(\mathrm{~m}, 5 \mathrm{H}), 1.58-1.71(\mathrm{~m}, 1 \mathrm{H}), 2.17(\mathrm{~d}, J=1.5 \mathrm{~Hz}, 3 \mathrm{H})$, $2.82(\mathrm{dt}, J=16.5$ and $2.7 \mathrm{~Hz}, 1 \mathrm{H}), 3.26(\mathrm{ddd}, J=16.5,8.4$, and $2.7 \mathrm{~Hz}, 1 \mathrm{H}), 4.08-4.19(\mathrm{~m}, 1 \mathrm{H}), 6.51(\mathrm{t}, J=2.7 \mathrm{~Hz}, 1 \mathrm{H})$, 7.06-7.46 (m, 5H); ${ }^{13} \mathrm{C} \mathrm{NMR}\left(\mathrm{CDCl}_{3}, 75 \mathrm{MHz}\right) \delta 9.18$, $13.91,22.53,26.63,31.17,32.52,70.80,122.43,127.35$, $128.59,128.72,135.30,136.54,146.23$.

Compound 5e: 36\%; oil; IR (neat) 2962, 2924, $1601 \mathrm{~cm}^{-1}$; ${ }^{1} \mathrm{H} \mathrm{NMR}\left(\mathrm{CDCl}_{3}, 300 \mathrm{MHz}\right) \delta 1.30(\mathrm{~s}, 6 \mathrm{H}), 2.20(\mathrm{~s}, 3 \mathrm{H})$, $2.71(\mathrm{~d}, J=2.7 \mathrm{~Hz}, 2 \mathrm{H}), 6.68(\mathrm{t}, J=2.7 \mathrm{~Hz}, 1 \mathrm{H}), 7.25-7.46$ $(\mathrm{m}, 5 \mathrm{H}) ;{ }^{13} \mathrm{C} \mathrm{NMR}\left(\mathrm{CDCl}_{3}, 75 \mathrm{MHz}\right) \delta 15.99,29.46,43.81$, 70.08, 125.38, 127.76, 128.53, 128.69, 136.88, 143.22, 168.84 .

Compound 6e: $46 \%$; white solid, $m p 87-89{ }^{\circ} \mathrm{C}$; IR (neat) 2974, 2931, 1543, $1265 \mathrm{~cm}^{-1} ;{ }^{1} \mathrm{H} \mathrm{NMR}\left(\mathrm{CDCl}_{3}, 300 \mathrm{MHz}\right) \delta$ 1.49 (s, 6H), 2.17 (s, 3H), 3.05 (d, $J=2.1 \mathrm{~Hz}, 2 \mathrm{H}), 6.52$ (t, $J$ $=2.1 \mathrm{~Hz}, 1 \mathrm{H}), 7.22-7.43(\mathrm{~m}, 5 \mathrm{H}) ;{ }^{13} \mathrm{C} \mathrm{NMR}\left(\mathrm{CDCl}_{3}, 75\right.$ MHz) $\delta 9.40,26.42,40.70,72.25,121.98,127.16,128.47$, $128.64,134.07,136.67,143.55$.

Typical procedures for the synthesis of Michael adduct 7a and bicyclic lactam derivative 9a: A solution of 5a (185 $\mathrm{mg}, 1 \mathrm{mmol})$ in methyl acrylate $(3 \mathrm{~mL})$ was heated to reflux for 4 days. After removal of methyl acrylate and column chromatographic purification process (hexanes/EtOAc, 2 : 1) we obtained $7 \mathbf{a}, 208 \mathrm{mg}(58 \%)$. To a stirred solution of $7 \mathbf{a}$ (179 mg, $0.5 \mathrm{mmol})$ in toluene $(3 \mathrm{~mL})$ was added $\mathrm{AcOH}$ (180 $\mathrm{mg}, 3 \mathrm{mmol})$ and the reaction mixture was heated to reflux for 3 days. After removal of solvent and column chromatographic purification process (hexanes $/ \mathrm{CH}_{2} \mathrm{Cl}_{2} / \mathrm{EtOAc}$, $2: 1: 2)$ we obtained $9 \mathbf{a}, 78 \mathrm{mg}(48 \%)$. The compounds $\mathbf{7 b}$, 7c, 8a, 9b, and 9c were synthesized analogously and the spectroscopic data are as follows.

Compound 7a: 58\%; oil; IR (neat) 2954, 1736, $1439 \mathrm{~cm}^{-1}$; ${ }^{1} \mathrm{H} \mathrm{NMR}\left(\mathrm{CDCl}_{3}\right) \delta 1.30(\mathrm{~d}, J=6.8 \mathrm{~Hz}, 3 \mathrm{H}), 1.95-2.50(\mathrm{~m}$, 
9H), 2.85-3.00 (m, 1H), 3.09 (ddd, $J=17.4,7.2$, and $2.7 \mathrm{~Hz}$, $1 \mathrm{H}), 3.65(\mathrm{~s}, 6 \mathrm{H}), 4.27-4.33(\mathrm{~m}, 1 \mathrm{H}), 6.75(\mathrm{t}, J=2.7 \mathrm{~Hz}$, $1 \mathrm{H})$, 7.26-7.49 (m, 5H); ${ }^{13} \mathrm{C} \mathrm{NMR}\left(\mathrm{CDCl}_{3}\right) \delta 22.84,28.48$, $31.36,31.52$, 35.95, 38.09, 51.53, 65.53, 124.62, 127.87, $128.57,128.93,136.81,142.63,173.82,175.40$; ESIMS $m / z$ $358.09\left(\mathrm{M}^{+}+\mathrm{H}\right)$.

Compound 7b: 28\%; IR (neat) 2958, 1736, $1169 \mathrm{~cm}^{-1} ;{ }^{1} \mathrm{H}$ NMR $\left(\mathrm{CDCl}_{3}, 300 \mathrm{MHz}\right) \delta 1.31(\mathrm{~s}, 6 \mathrm{H}), 1.90-2.17(\mathrm{~m}, 4 \mathrm{H})$, 2.22-2.47 (m, 4H), $2.73(\mathrm{~d}, J=2.7 \mathrm{~Hz}, 2 \mathrm{H}), 2.89-2.95(\mathrm{~m}$, $1 \mathrm{H}), 3.64(\mathrm{~s}, 6 \mathrm{H}), 6.73(\mathrm{t}, J=2.7 \mathrm{~Hz}, 1 \mathrm{H}), 7.20-7.47(\mathrm{~m}$, $5 \mathrm{H}) ;{ }^{13} \mathrm{C}$ NMR $\left(\mathrm{CDCl}_{3}, 75 \mathrm{MHz}\right) \delta 28.54,29.56,31.39$, $35.76,44.33,51.49,70.33,124.86,127.85,128.54,128.91$, $136.82,142.92,173.13,173.78$.

Compound 7c: $45 \%$ (1:1 mixture of two diastereomers); IR (neat) 2962, $1736 \mathrm{~cm}^{-1} ;{ }^{1} \mathrm{H}$ NMR $\left(\mathrm{CDCl}_{3}, 300 \mathrm{MHz}\right) \delta$ $1.25(\mathrm{~d}, J=6.9 \mathrm{~Hz}, 1.5 \mathrm{H}), 1.26(\mathrm{~d}, J=6.9 \mathrm{~Hz}, 1.5 \mathrm{~Hz}), 1.29$ $(\mathrm{d}, J=6.9 \mathrm{~Hz}, 1.5 \mathrm{H}), 1.30(\mathrm{~d}, J=6.9 \mathrm{~Hz}, 1.5 \mathrm{~Hz}, 1.5 \mathrm{H})$, $1.85-1.95(\mathrm{~m}, 1 \mathrm{H}), 2.08-2.22(\mathrm{~m}, 1 \mathrm{H}), 2.28-2.48(\mathrm{~m}, 3 \mathrm{H})$, 2.90-2.97 (m, $1 \mathrm{H}$ ), 3.07 (ddd, $J=17.4,6.9$, and $2.4 \mathrm{~Hz}, 1 \mathrm{H}$ ), $3.66(\mathrm{~s}, 3 \mathrm{H}), 4.24-4.30(\mathrm{~m}, 1 \mathrm{H}), 6.75(\mathrm{t}, J=2.4 \mathrm{~Hz}, 1 \mathrm{H})$, 7.25-7.48 (m, 5H); ${ }^{13} \mathrm{C}$ NMR $\left(\mathrm{CDCl}_{3}, 75 \mathrm{MHz}\right) \delta 18.94$, $19.10,22.67,22.82,29.96,30.12,31.59,31.69,31.77$, $38.12,38.19,51.46,65.30,65.41,124.14,124.22,127.68$, $128.53,128.83,136.98,142.04,174.01,176.86,177.00$.

Compound 8a: 59\%; oil; IR (neat) 2927, 2245, $1589 \mathrm{~cm}^{-1}$; ${ }^{1} \mathrm{H}$ NMR $\left(\mathrm{CDCl}_{3}, 300 \mathrm{MHz}\right) \delta 1.32(\mathrm{~d}, J=6.6 \mathrm{~Hz}, 3 \mathrm{H})$, 1.92-2.05 (m, 2H), 2.14-2.55 (m, 7H), 3.10-3.23 (m, 2H), 4.28-4.38 (m, 1H), $6.87(\mathrm{t}, J=2.7 \mathrm{~Hz}, 1 \mathrm{H}), 7.22-7.51(\mathrm{~m}$, $5 \mathrm{H}) ;{ }^{13} \mathrm{C}$ NMR $\left(\mathrm{CDCl}_{3}, 75 \mathrm{MHz}\right) \delta 14.81,14.98,22.66$, $29.12,35.21,38.03,65.81,119.21,125.43,128.33,128.67$, 129.13, 136.16, 141.99, 173.39; ESIMS $m / z 292.10\left(\mathrm{M}^{+}+\mathrm{H}\right)$.

Compound 9a: 48\%; oil; IR (neat) 2954, 1732, $1666 \mathrm{~cm}^{-1}$; ${ }^{1} \mathrm{H}$ NMR $\left(\mathrm{CDCl}_{3}, 300 \mathrm{MHz}\right) \delta 1.17(\mathrm{~d}, J=6.3 \mathrm{~Hz}, 3 \mathrm{H})$, 2.22-2.60 (m, 7H), 2.66-2.89 (m, 2H), 2.95-3.04 (m, 1H), $3.70(\mathrm{~s}, 3 \mathrm{H}), 4.37-4.48(\mathrm{~m}, 1 \mathrm{H}), 6.90(\mathrm{~s}, 1 \mathrm{H}), 7.24-7.41(\mathrm{~m}$, $5 \mathrm{H}) ;{ }^{13} \mathrm{C}$ NMR $\left(\mathrm{CDCl}_{3}, 75 \mathrm{MHz}\right) \delta 20.04,26.74,27.95$, $30.86,32.79,36.57,50.98,51.74,112.17,126.84,127.25$, $128.35,128.92,133.93,134.72,137.00,167.59,173.16$.

Compound 9b: 40\%; oil; IR (neat) 2924, 1736, $1651 \mathrm{~cm}^{-1}$; ${ }^{1} \mathrm{H}$ NMR $\left(\mathrm{CDCl}_{3}, 300 \mathrm{MHz}\right) \delta 1.49$ (s, 6H), 2.30-2.37 (m, $2 \mathrm{H}), 2.40-2.47(\mathrm{~m}, 2 \mathrm{H}), 2.52-2.59(\mathrm{~m}, 2 \mathrm{H}), 2.71-2.77(\mathrm{~m}$, $4 \mathrm{H}), 3.69(\mathrm{~s}, 3 \mathrm{H}), 6.78(\mathrm{~s}, 1 \mathrm{H}), 7.19-7.41(\mathrm{~m}, 5 \mathrm{H}) ;{ }^{13} \mathrm{C} \mathrm{NMR}$ $\left(\mathrm{CDCl}_{3}, 75 \mathrm{MHz}\right) \delta 25.72,26.08,28.05,32.69,33.15,45.54$, $51.77,61.39,112.44,126.14,127.22,128.37,128.96$, $132.75,136.70,137.01,168.18,173.25$.

Compound 9c: 58\%; oil; IR (neat) $1651,1404 \mathrm{~cm}^{-1} ;{ }^{1} \mathrm{H}$ $\operatorname{NMR}\left(\mathrm{CDCl}_{3}, 300 \mathrm{MHz}\right) \delta 1.18(\mathrm{~d}, J=6.3 \mathrm{~Hz}, 3 \mathrm{H}), 2.08(\mathrm{~s}$, $3 \mathrm{H}), 2.26-2.35(\mathrm{~m}, 1 \mathrm{H}), 2.45-2.58(\mathrm{~m}, 4 \mathrm{H}), 3.00$ (ddd, $J=$ $15.3,8.7$, and $3.3 \mathrm{~Hz}, 1 \mathrm{H}), 4.39-4.50(\mathrm{~m}, 1 \mathrm{H}), 6.84(\mathrm{~s}, 1 \mathrm{H})$, 7.22-7.40 (m, 5H); ${ }^{13} \mathrm{C} \mathrm{NMR}\left(\mathrm{CDCl}_{3}, 75 \mathrm{MHz}\right) \delta 19.37$, $20.11,29.58,30.75,36.45,50.93,109.83,126.98,127.03$, $128.34,128.77,133.36,134.67,137.29,167.47$.

Acknowledgements. This work was supported by the grant (R-05-2003-000-10042-0) from the Basic Research Program of the Korea Science and Engineering Foundation
(Now controlled under the authority of Korea Research Foundation). Spectroscopic data was obtained from the Korea Basic Science Institute, Gwangju branch.

\section{References and Notes}

1. Basavaiah, D.; Rao, J. S. Tetrahedron Lett. 2004, 45, 1621.

2. For the reductive cyclization of $\gamma$-nitrocarbonyl compounds, see (a) Black, D. St. C.; Edwards, G. L.; Evans, R. H.; Keller, P. A.; Laaman, S. M. Tetrahedron 2000, 56, 1889. (b) Taniguchi, M.; Ra, D.; Mo, G.; Balasubramanian, T.; Lindsey, J. S. J. Org. Chem. 2001, 66, 7342. (c) Nsanzumuhire, C.; Clement, J.-L.; Ouari, O.; Karoui, H.; Finet, J.-P.; Tordo, P. Tetrahedron Lett. 2004, 45, 6385. (d) Ningsanont, N.; Black, D. St. C.; Chanphen, R.; Thebtaranonth, Y. J. Med. Chem. 2003, 46, 2397. (e) Turner, M. J.; Luckenbach, L. A.; Turner, E. L. Synth. Commun. 1986, 16, 1377. (f) Halland, N.; Hazell, R. G.; Jorgensen, K. A. J. Org. Chem. 2002, 67, 8331. (g) Cariou, M.; Jubault, H. M.; Tallec, et A. Tetrahedron Lett. 1982, 22, 3961. (h) Cheruku, S. R.; Padmanilayam, M. P.; Vennerstrom, J. L. Tetrahedron Lett. 2003, 44, 3701.

3. For the synthesis and synthetic applications of pyrrolines, see (a) van Esseveldt, B. C. J.; Vervoort, P. W. H.; van Delft, F. L.; Rutjes, F. P. J. T. J. Org. Chem. 2005, 70, 1791. (b) Kitamura, M.; Mori, Y.; Narasaka, K. Tetrahedron Lett. 2005, 46, 2373. (c) Gawley, R. E.; Termine, E. J. J. Org. Chem. 1984, 49, 1946.

4. For the synthesis and synthetic applications of pyrroline $\mathrm{N}$-oxides, see (a) Black, D. St. C.; Craig, D. C.; Edwards, G. L.; Laaman, S. M. Tetrahedron Lett. 1998, 39, 5849. (b) Desvergnes, S.; Py, S.; Vallee, Y. J. Org. Chem. 2005, 70, 1459.

5. For our recent publications on the chemical transformations of Baylis-Hillman adducts, see (a) Lee, C. G.; Lee, K. Y.; Kim, S. C.; Kim, J. N. Bull. Korean Chem. Soc. 2005, 26, 485. (b) Lee, C. G; Gowrisankar, S.; Kim, J. N. Bull. Korean Chem. Soc. 2005, 26, 481. (c) Lee, M. J.; Lee, K. Y.; Kim, J. N. Bull. Korean Chem. Soc. 2005, 26, 477. (d) Gowrisankar, S.; Na, J. E.; Lee, M. J.; Kim, J. N. Bull. Korean Chem. Soc. 2005, 26, 319. (e) Lee, C. G.; Lee, K. Y.; Lee, S.; Kim, J. N. Tetrahedron 2005, 61, 1493. (f) Lee, K. Y.; Kim, T. H.; Kim, J. N. Bull. Korean Chem. Soc. 2004, 25, 1966. (g) Gowrisankar, S.; Lee, C. G.; Kim, J. N. Tetrahedron Lett. 2004, 45, 6949. (h) Gowrisankar, S.; Lee, K. Y.; Lee, C. G.; Kim, J. N. Tetrahedron Lett. 2004, 45, 6141. (i) Lee, K. Y.; Gowrisankar, S.; Kim, J. N. Tetrahedron Lett. 2004, 45, 5485. (j) Kim, J. M.; Lee, K. Y.; Lee, S.-k.; Kim, J. N. Tetrahedron Lett. 2004, 45, 2805.

6. For the Michael type reaction of imine compounds, see (a) Monnier-Benoit, N.; Jabin, I.; Selkti, M.; Tomas, A.; Revial, G. Tetrahedron: Asymmetry 2003, 14, 2747. (b) Camara, C.; Keller, L.; Dumas, F. Tetrahedron: Asymmetry 2003, 14, 3263. (c) d'Angelo, J.; Guingant, A. Tetrahedron Lett. 1988, 29, 2667. (d) Desmaele, D.; d'Angelo, J. Tetrahedron Lett. 1989, 30, 345. (e) Pfau, M.; Revial, G.; Guingant, A.; d'Angelo, J. J. Am. Chem. Soc. 1985, 107, 273

7. For the imine-enamine tautomerization of 2-methylpyrroline derivatives, see reference $2 b$.

8. (a) Aube, J.; Milligan, G. L. J. Am. Chem. Soc. 1991, 113, 8965. (b) Milligan, G. L.; Mossman, C. J.; Aube, J. J. Am. Chem. Soc. 1995, 117, 10449. (c) Sato, Y.; Nukui, S.; Sodeoka, M.; Shibasaki, M. Tetrahedron 1994, 50, 371. (d) Nukui, S.; Sodeoka, M.; Shibasaki, M. Tetrahedron Lett. 1993, 34, 4965. (e) Nukui, S.; Sodeoka, M.; Sasai, H.; Shibasaki, M. J. Org. Chem. 1995, 60, 398. (f) Mori, M.; Hashimoto, A.; Shibasaki, M. J. Org. Chem. 1993, 58, 6503. (g) Schumann, D.; Naumann, A.; Wirtz, K.-P Chem. Ber. 1979, 112, 734. (h) Padwa, A.; Sheehan, S. M.; Straub, C. S. J. Org. Chem. 1999, 64, 8648. (i) Takahata, H.; Takamatsu, T.; Yamazaki, T. J. Org. Chem. 1989, 54, 4812.

9. Maison, W.; Prenzel, A. H. G. P. Synthesis 2005, 1031. 